\title{
The value of plasma pro-enkephalin and adrenomedullin for the prediction of sepsis-associated acute kidney injury in critically ill patients
}

\author{
Ruijin $\mathrm{Liu}^{1+}$, Xiaoya Zheng ${ }^{1 \dagger}$, Hongliang Wang ${ }^{2 \dagger}$, Sicong Wang ${ }^{1}$, Kaijiang $\mathrm{Yu}^{3^{*}}$ and Changsong Wang ${ }^{{ }^{*}}$
}

Kidney function is commonly affected by sepsis, which is one of the most prominent causes of acute kidney injury (AKI) [1]. Seeking early, reliable biomarkers for the detection of AKI in critically ill patients with sepsis, it is of great importance to conduct successful interventions and reduce adverse outcomes.

Pro-enkephalin is considered as a reliable alternative marker of enkephalin, which is an endogenous opioid peptide and may be associated with decreased renal function [2]. Adrenomedullin, a protein from the calcitonin family, has been reported to increase significantly in patients with sepsis [3]. We prospectively evaluated the predictive value of plasma pro-enkephalin and adrenomedullin (Shuwen Biotech Co. Ltd, China) levels for septic AKI compared to other candidate biomarkers (neutrophil gelatinase-associated lipocalin (NGAL), cystatin-C, kidney injury molecule 1 (KIM-1), and interleukin 18 (IL-18)) (Boster Biological Technology co. Ltd, China).

Forty-two septic patients (age $\geq 18$ ) in the intensive care unit were included in the final analysis. Blood samples were collected from a peripheral vein or central venous within $24 \mathrm{~h}$ after the patient was diagnosed with sepsis based on sepsis-3 criteria [4]. According to the 2013 KDIGO standard, patients were divided into one of the cohorts and staged based on the worst serum creatinine and/or the lowest urine output [5].

\footnotetext{
*Correspondence: drkaijiang@163.com; changsongwangicu@163.com ${ }^{+}$Ruijin Liu, Xiaoya Zheng, and Hongliang Wang contributed equally. ${ }^{3}$ Department of Critical Care Medicine, The First Affiliated Hospital of Harbin Medical University, No. 23 Youzheng Rd., Nangang District, Harbin 150001, China

'Department of Critical Care Medicine, Harbin Medical University Cancer Hospital, No. 150 Haping Rd., Nangang District, Harbin 150081, China Full list of author information is available at the end of the article
}

Among the participants, sixteen patients subsequently suffered from AKI, five of whom were in stage 1, eleven were in stage 3 , and no patients were in stage 2 . A significant difference of plasma pro-enkephalin concentration exists in the septic AKI group and sepsis group (median 229.2 (93.62-341.2) vs $64.71(49.23-90.87) \mathrm{pmol} / \mathrm{L}, P<0.0001)$. In addition, patients in AKI stage 3 had higher plasma proenkephalin $(292.94 \pm 140.18$ vs $125.06 \pm 65.82 \mathrm{pg} / \mathrm{mL})$ levels than those in AKI stage 1. The plasma adrenomedullin concentration in the septic AKI group was significantly higher than that in the sepsis group (median 164.69 (118.07-193.52) vs $76.5(48.66-132.31) \mathrm{pg} / \mathrm{mL}, P=0.0229)$. There was no significant difference in plasma NGAL (median 2.37(2.21-2.37) vs $2.25(1.96-3.32) \mathrm{pg} / \mathrm{mL}, P=0.9631)$, cystatin-C (mean $28.85 \pm 7.25$ vs $31.24 \pm 14.72 \mathrm{pg} / \mathrm{mL}, P=$ 0.7353), KIM-1 (median 408.21(0.91-666.5) vs 96.22(0.26260.12) $\mathrm{pg} / \mathrm{mL}, \quad P=0.1795$ ), and IL-18 (median $136.1(133.36-524.19)$ vs $184.71(101.84-665.98) \mathrm{pg} / \mathrm{mL}, P=$ 0.0229 ) existed in septic patients with and without AKI.

The receiver operating characteristic analysis for the assessment of the diagnostic accuracy of pro-enkephalin and adrenomedullin in the prediction of AKI in septic patients showed significant predictive value for both biomarkers, with area under curve (AUC) of 0.884 (95\% CI, $0.738-0.965)$ and 0.731 (95\% CI, 0.560-0.863), respectively (Fig. 1). Pro-enkephalin had a sensitivity of $60.87 \%$ and specificity of $100 \%$ at the cutoff value of $66.97 \mathrm{pmol} /$ L, while adrenomedullin had a sensitivity and specificity of $75 \%$ and $76.92 \%$, respectively, at a cutoff value of $110.44 \mathrm{pg} / \mathrm{mL}$. The combination of the two biomarkers revealed the highest discrimination and had an AUC of 0.890 (95\% CI, 0.740-0.969) as well as a sensitivity of $92.31 \%$ and a specificity of $69.57 \%$. The pro-enkephalin 

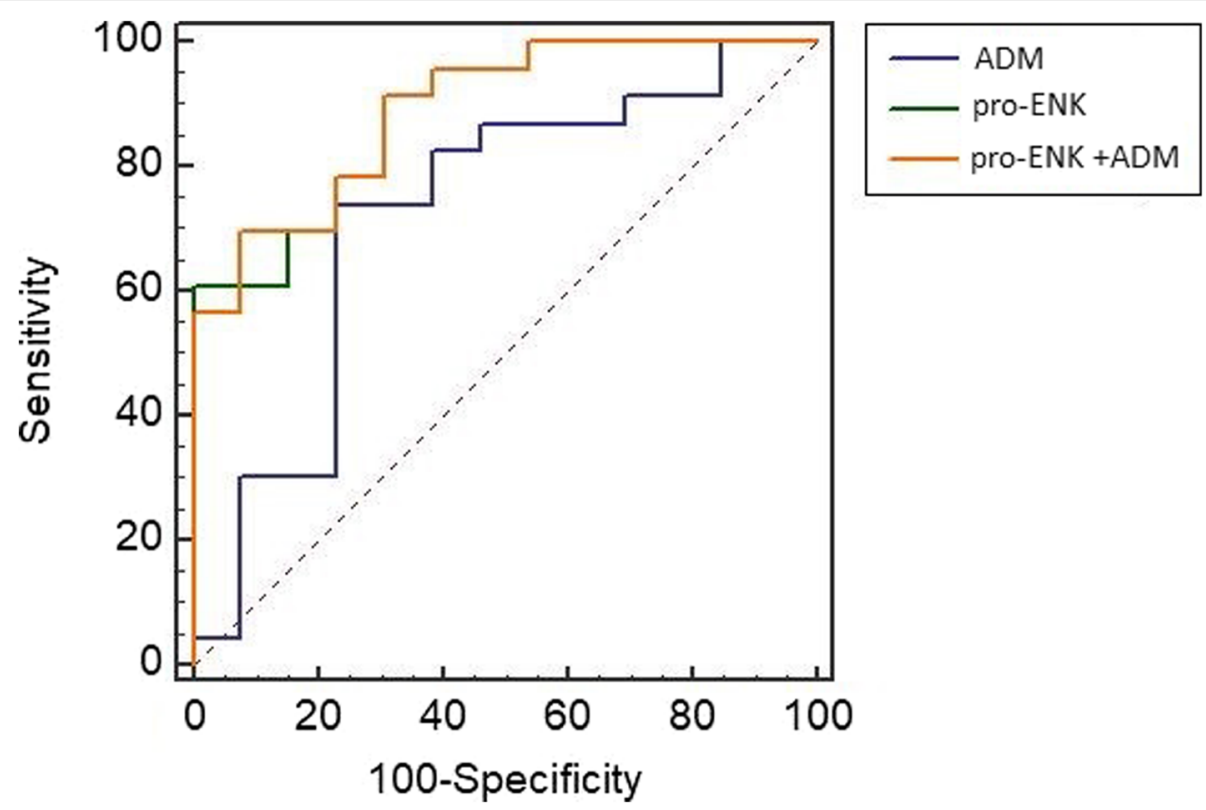

Fig. 1 The receiver operating characteristic curve showing the diagnostic power of pro-enkephalin, adrenomedullin, and the combination of proenkephalin and adrenomedullin for the estimation of acute kidney injury in septic patients. pro-ENK, pro-enkephalin; ADM, adrenomedullin

and adrenomedullin are promising biomarkers for physicians to promptly assess the presence and severity of AKI at an early stage, which will make contribution to start adequate treatment immediately and avoid worse outcome. The limitations of our study include the limited sample size as well as the certain biomarkers, such as NGAL, KIM-1, and IL-18, lacking the detection data of urine specimens which may differ from the diagnostic accuracy of blood specimens.

\section{Acknowledgements}

Not applicable.

\section{Authors' contributions}

LRJ and WSC conducted sample collection and biochemical analysis. ZXY and WHL were involved in the data collection and statistical analysis. LRJ and ZXY contributed to data interpretation and drafting of the manuscript. WCS and YKJ contributed to study design, manuscript revision, and providing facilities. The authors read and approved the final manuscript.

\section{Funding}

KJY was funded by the National Natural Science Foundation of China (No.81571871 and 81770276) and Nn10 program. CSW was supported by Distinguished Young Scholars Fund of Harbin Medical University Cancer Hospital, the Yuweihan Fund for Distinguished Young Scholars of Harbin Medical University, Harbin Science and Technology Innovation Scholar Fund (2017RAXXJ087), and Heilongjiang Province Postdoctoral Research Fund. The funding sources were not involved in the design of the study; in the collection, analysis, and interpretation of data; or in writing the manuscript

\section{Availability of data and materials}

Data are available on request.

\section{Ethics approval and consent to participate}

The study protocol was approved by the ethical committee of the Second Affiliated Hospital of Harbin Medical University (Harbin, China) (KY2018-292). Written informed consent was obtained from all patients or their surrogates.

\section{Consent for publication}

Not applicable.

\section{Competing interests}

The authors declare that they have no competing interests.

\section{Author details}

'Department of Critical Care Medicine, Harbin Medical University Cancer Hospital, No. 150 Haping Rd., Nangang District, Harbin 150081, China.

${ }^{2}$ Department of Critical Care Medicine, The Second Affiliated Hospital of Harbin Medical University, Harbin, China. ${ }^{3}$ Department of Critical Care Medicine, The First Affiliated Hospital of Harbin Medical University, No. 23 Youzheng Rd., Nangang District, Harbin 150001, China.

Received: 23 February 2020 Accepted: 13 April 2020

Published online: 21 April 2020

\section{References}

1. Murugan R, Karajala-Subramanyam V, Lee M, Yende S, Kong L, Carter M, et al. Acute kidney injury in non-severe pneumonia is associated with an increased immune response and lower survival. Kidney Int. 2010;77(6):527-35.

2. Arbit B, Marston N, Shah K, Lee EL, Aramin H, Clopton P, et al. Prognostic usefulness of proenkephalin in stable ambulatory patients with heart failure. Am J Cardiol. 2016;117(8):1310-4.

3. Bernal-Morell E, García-Villalba E, Vera MDC, Medina B, Martinez M, Callejo V, et al. Usefulness of midregional pro-adrenomedullin as a marker of organ damage and predictor of mortality in patients with sepsis. J Inf Secur. 2018; 76(3):249-57.

4. Singer M, Deutschman CS, Seymour CW, Shankar-Hari M, Annane D, Bauer $M$, et al. The third international consensus definitions for sepsis and septic shock (Sepsis-3). JAMA. 2016;315(8):801-10.

5. Kellum JA, Lameire N, KDIGO AKI Guideline Work Group. Diagnosis, evaluation, and management of acute kidney injury: a KDIGO summary (Part 1). Crit Care. 2013;17(1):204

\section{Publisher's Note}

Springer Nature remains neutral with regard to jurisdictional claims in published maps and institutional affiliations. 